\title{
Scaled beam merging experiment for heavy ion inertial fusion
}

\author{
P. A. Seidl, ${ }^{*}$ C. M. Celata, A. Faltens, and E. Henestroza \\ Lawrence Berkeley National Laboratory, Berkeley, California 94720, USA
}

\section{S. A. MacLaren}

Lawrence Livermore National Laboratory, Livermore, California 94550, USA

(Received 12 June 2003; published 12 September 2003)

\begin{abstract}
Transverse beam combining is a cost-saving option employed in many designs for heavy ion fusion drivers. However, the resultant transverse phase space dilution must be minimized so as not to sacrifice focusability at the target. A prototype combining experiment has been completed employing four 3-mA $\mathrm{Cs}^{+}$beams injected at $160 \mathrm{keV}$. The focusing elements upstream of the merge consist of four quadrupoles and a final combined-function element (quadrupole and dipole). Following the merge, the resultant single beam is transported in a single alternating gradient channel where the subsequent evolution of the distribution function is diagnosed. The results are in fair agreement with particle-incell simulations. They indicate that for some heavy ion fusion driver designs, the phase space dilution from merging is acceptable.
\end{abstract}

DOI: 10.1103/PhysRevSTAB.6.090101

PACS numbers: 52.59.Sa, 41.85.Ne, 52.59.Sa, 52.65.Rr

\section{BACKGROUND}

In heavy ion driven fusion, the stored energy from the ion beam driver is used to heat, compress, and ignite inertially confined fusion fuel. The target and reactor chamber requirements set the incident beam parameters and thus the driver requirements. The required incident energy is $1-10 \mathrm{MJ}$ (for deuterium-tritium targets) delivered within $\sim 10 \mathrm{~ns}$, corresponding to $10^{14}-10^{15} \mathrm{~W}$ of peak beam power. The U.S. community has been exploring induction-based accelerators, with the baseline approach a linear-induction accelerator. The driver has multiple beams $(100-200)$ in a transverse array that threads common induction cores for acceleration. The need to hit a small target at the chamber center using lenses at $\sim 6 \mathrm{~m}$ distance sets the maximum phase space requirement on each of the beams. Six-dimensional phase space volume (which determines focusability at the target), limitations of focusing high space-charge beams, and the required beam edge-to-aperture clearance (fill factor) are considerations that determine the actual number of beams in the most cost-effective, technically sound driver design.

The aperture fill factor is the ratio of the maximum beam-envelope radius to the beam pipe radius. High fill factors exceeding $50 \%$ are desirable to minimize the focusing array size and in turn the amount of induction core material needed for economical acceleration, but high fill factors also increase the probability of undesirable beam-wall interactions. Further limitations on the fill factor and transportable current are beam centroid oscillations due to alignment imperfections, envelope mismatch, and the deterioration of focusing field quality

*Electronic address: PASeidl@lbl.gov at large radii due to higher multipole components in the body of the lens and its ends. The six-dimensional phase space volume of each of the beams emerging from the sources can be lower by a factor $>10^{2}$ than what it required for focusing at the target. Processes leading to emittance growth must be understood and with this understanding, a most cost-effective driver will take advantage of this available emittance budget through trade-offs in precision of construction and advantageous beam manipulations.

One such beam manipulation option is the transverse merging of four beams (from separate beam lines) into a single beam line. It is of interest when considering a beam line employing both electrostatic quadrupoles and superconducting magnetic quadrupoles for focusing the beams along the $3-5 \mathrm{~km}$ long driver. Magnetic quadrupoles have a greater beam focusing strength (than electrostatic quadrupoles) with increasing beam velocity while electrostatic quadrupoles may be preferable where short lattice periods are required at lower beam energy. Electrostatic focusing is also advantageous at low kinetic energy, since secondary electrons from beam-wall and beambackground gas interactions (building up during the initial $\tau_{p} \approx 30 \mu$ s driver beam pulse) would be swept out of the beam path by the quadrupole focusing field. A relatively high fractional cost of the accelerator is due to the induction cores, and the core material is minimized in a design with the lowest transverse cross-sectional area of the multibeam focusing arrays. Thus, it is important to maximize the beam current density over the cross section of the array (i.e., the ratio [sum of all beams]:[cross-sectional area of the array]). The optimum number of channels and the size of each channel is linked to beam properties (e.g., halo) and the choice of focusing technology. The dimensions of electrostatic quadrupoles (based 
on an array of cylindrical electrodes) will be constrained by the highest electric field achievable without breakdown, field quality specifications, and beam edge to electrode clearance. Similarly an array of superconducting magnetic quadrupoles will be constrained by the critical field and current density of the coils, coil dimensions, cryostat dimensions, field quality specifications, and beam edge to electrode clearance. Because of the larger transverse thickness of the magnetic quadrupole hardware, it tends to be more advantageous to use magnetic quadrupoles where the beam is large, so that beam clearances and conductor dimensions do not dominate the cross-sectional area in the array. Since the amount of transportable current for a given focusing strength scales as the beam energy, this implies that magnetic quadrupoles would be most advantageous at higher energies and electrostatic at lower energy. There are design solutions for heavy ion injectors at $\approx 2 \mathrm{MV}$ which produce $\sim 1 \mathrm{~A}$ of beam. Magnetic channels optimize at substantially higher current. The current in an induction linac can be increased by compressing the single bunch as it travels along the machine, but this requires a length of accelerator and associated cost. Another option, the subject of this paper, is merging the beams to produce fewer, higher current beams in a short distance.

An advantageous transition from electrostatic to magnetic focusing might occur at $\approx 100 \mathrm{MeV}$, by merging four beams from the electrostatic section into a single magnetic-focused beam line. The number of beams that then must be accelerated and transported through common induction cores is $1 / 4$ the initial number, each with $4 \times$ the premerge beam current. The current of the merged beams is then well matched to the optimum average beam current density transportable through arrays of superconducting magnetic quadrupoles. The energy at which to merge beams is dictated by halo particle loss for long pulses (20-30 $\mu \mathrm{s})$ at low kinetic energy, economics, and the point at which magnetic quadrupole focusing becomes more effective. When the beam kinetic energy is $100 \mathrm{MeV}$, the pulse length has greatly decreased (2-4 $\mu \mathrm{s})$, which decreases the impact of halo-induced gas desorption on the latter part of the beam pulse. In magnetic quadrupoles the shorter pulse length limits the unwanted buildup or trapping of secondary electrons in the beam potential. Beam loss due to merging will not lead to activation, since the ion energy is still below the threshold for overcoming the nucleus-nucleus Coulomb barrier.

There is analogous research to the beam merging studied in this experiment. Multiturn injection is common in high-energy synchrotron and storage rings. In the rf accelerator approach to heavy ion fusion (HIF) [1], the required beam current cannot be produced from a single ion source. After the multiple beam ion injector the beam current is increased by funneling (i.e., longitudinal merging) pairs of beams into a single accelerator beam line [2-4] with a corresponding doubling of rf frequency.
The current increase comes from packing bunches more densely along the beam axis, and four 2:1 stages of funneling are required to achieve the required current (400 m A). To keep the emittance of the final beam small, a challenge to this beam manipulation is to maintain nearly identical beam parameters for the funnel beams so that they overlap in phase space as much as possible.

In another recent example, transverse merging of many $(\approx 100) 2$ - $\mathrm{mm}$ diameter beams each with $\approx 5 \mathrm{~mA}$ and $\approx 20 \mu$ s duration has been proposed for injection into alternating-gradient channels of induction-linac heavy ion fusion drivers [5]. In that case the merging occurs at $1 \mathrm{MeV}$ in an accelerating column with $100 \mathrm{kV} / \mathrm{cm}$, with further acceleration to $1.6 \mathrm{MeV}$ before injection into the rest of the accelerator. The objective is to keep the emittance growth small. Thus, mechanical tolerances are tight, and desorption and beam-gas interactions are a concern with the edge of the $2-\mathrm{mm}$ beam only $1 \mathrm{~mm}$ from the $4 \mathrm{~mm}$ aperture boundary.

In the transverse beam merging process described here (and in [5]), the free space-charge energy of the configuration of beams in the initial state of the merge leads to emittance growth. This is a significant effect, since the generalized perveance of the beams before the merge is considerable, $K=q I / 2 \pi \varepsilon_{0} m v^{3} \approx 10^{-4}$, and the initial state charge distribution is highly nonuniform [6]. The nonlinear space-charge field launches transverse modes of oscillation because the beam distribution is not in equilibrium with the applied and self-fields. These collective modes phase mix and relax in a distance that is roughly characterized by the plasma frequency of the beam. Single particle dynamics associated with the wrapping up of empty regions in phase space between the merging beams also contributes to the emittance of the equilibrated final (merged) state. An analytical description of the emittance growth from merging arrays of beams is in [6,7]. Early results from this experiment are in [8-10]. Overviews of the U.S. heavy ion driven inertial fusion energy research program are in $[11,12]$ and a review of the main classes of architecture for heavy ion induction accelerator drivers is in [13].

\section{DESCRIPTION OF THE EXPERIMENT}

At Lawrence Berkeley National Laboratory an experiment to demonstrate 4-to-1 transverse beam combining has been built using the old multiple-beam experiment (MBE-4) accelerator [14]. The MBE-4 apparatus was used mainly to provide a Marx-based injector and a long transport channel (of 62 electrostatic quadrupoles) for measuring phase space evolution; the "beam combiner "is new. The combiner system, consisting of four $\mathrm{Cs}^{+}$ sources, each followed by a $160 \mathrm{kV}$ diode and a focusing transport channel, replaced the old MBE-4 diode and matching system. The four beam lines (including the sources and diodes) initially converge with an angle 


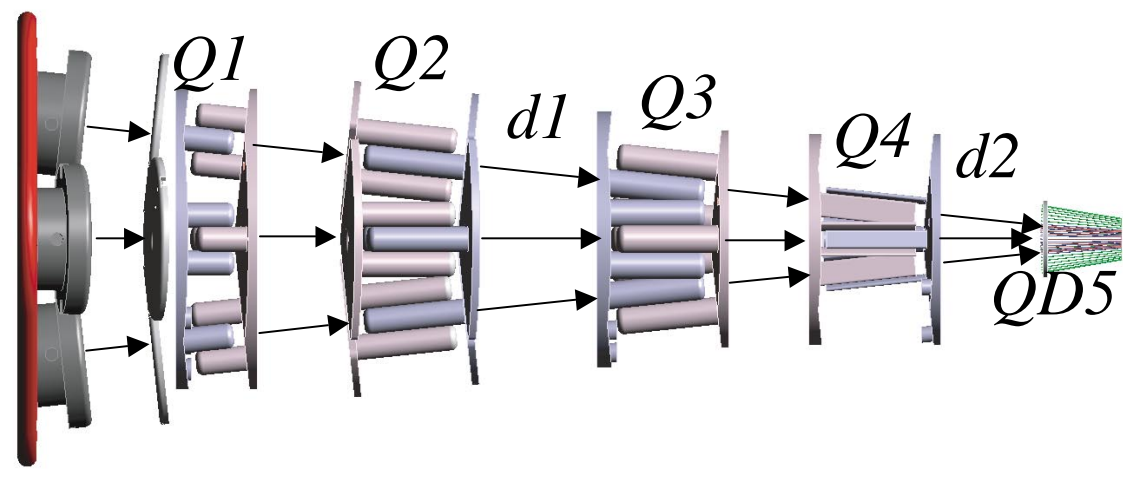

FIG. 1. (Color) A CAD view of the lattice elements of the Combiner apparatus. Diagnostics are at locations marked $d 1$ and $d 2$. The first four elements $(Q 1-Q 4)$ are electrostatic quadrupoles. Each of the quadrupoles of $Q 2$ is separately moveable in both horizontal and vertical dimensions. $Q D 5$ is the combined function dipole and quadrupole, for which the electrostatic field-defining rods around each beam converge as $z$ increases. The distance from the source emitting surfaces to the end of $Q D 5$ is $\approx 108 \mathrm{~cm}$.

of $6^{\circ}$ relative to the combiner centerline. This scaled experiment does not include the initial dipole elements that would begin to merge the beams from the upstream multiple beam array of a driver, a simplification of the experiment compared to a driver-scale combiner, which would not significantly alter the experiment. The four arrays of electrostatic quadrupoles $(Q 1-Q 4)$, followed by an electrostatic combined-function (quadrupole and dipole) element (QD5), focus each beam and then straighten its trajectory relative to the centerline just before the merge, so that the beams emerge from the combiner parallel to the centerline of the MBE-4 transport channel. A side view of the principal elements of the combiner apparatus is shown in Fig. 1. The design configurations for the beam cross sections as they emerge from the combiner are shown in Fig. 2. As can be seen, the cross section is $x-y$ asymmetric to allow for good packing of the elliptical beams. The associated transverse emittance ellipses are upright in phase space in order to further minimize phase space dilution. After the combiner, the merged beam is transported (without acceleration) and diagnosed in the remaining 31 lattice periods of one channel of MBE-4 (Q6-Q67). This is a long enough lattice to allow the merged beam to equilibrate. Typical operating voltages are shown in Table I.

Q4 has hyperbolic-shaped electrodes, because the closer proximity of the beam trajectories to one another did not allow for cylindrical electrodes large enough to provide good field quality between the beams. The quadrupole and dipole fields in $Q D 5$ are produced by a "wire cage" of 1-mm diameter straight tungsten rods at a spacing of $\sim 2 \mathrm{~mm}$, approximately parallel to the beams' paths. Seventy-one such wires are used in the complete cage to individually focus the beams. The voltage on each rod is set according to the desired Dirichlet boundary condition. This results in voltage differences of up to $1.5 \mathrm{kV}$ from rod to rod. The rods are cantilevered from a ceramic support plate and potentials are set on the rods

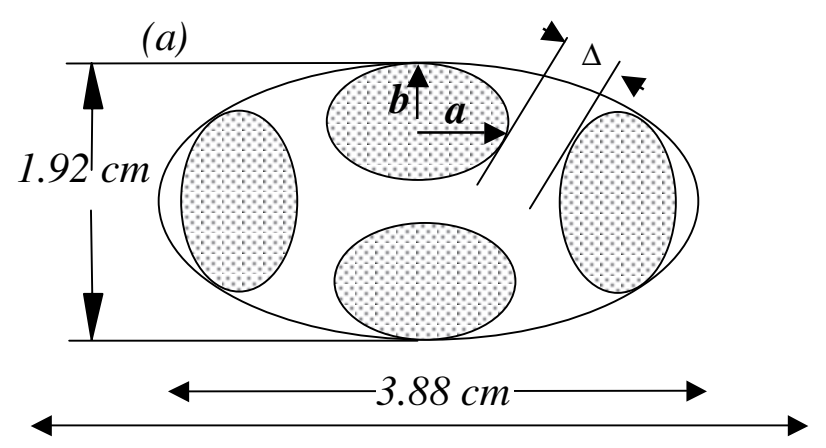

Downstream transport aperture $=5.4 \mathrm{~cm}$

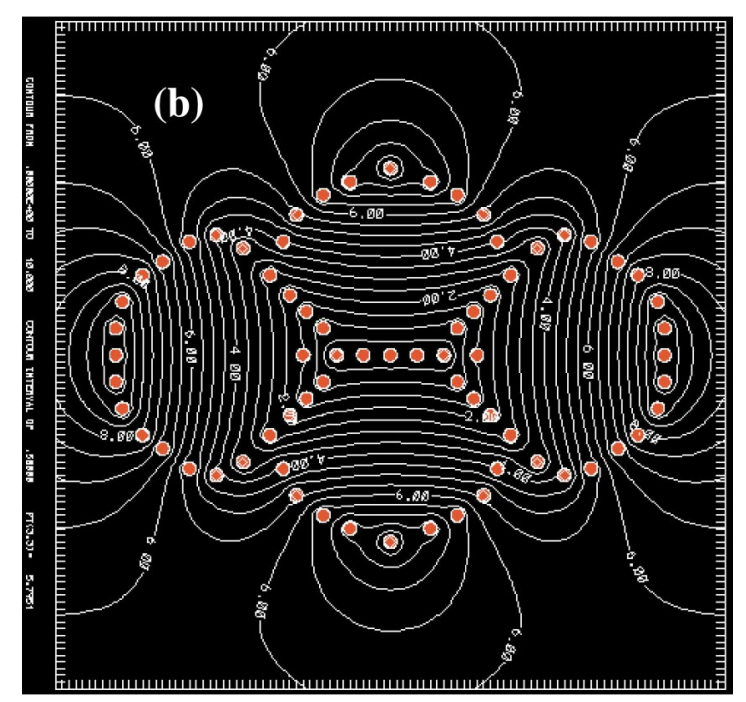

FIG. 2. (Color) (a) Schematic of the beam footprints at the exit of $Q D 5$. The individual beam semimajor and semiminor axes are 6.5 and $3.7 \mathrm{~mm}$. The beam edge to beam-edge clearance is $\Delta=4 \mathrm{~mm}$. The upstream matching solution is chosen so that the beam envelope divergence angles are zero $\left(a^{\prime}=b^{\prime}=0\right)$ at this point, which also helps to minimize phase space dilution of the merged beam. (b) The dipole component is apparent from the equipotentials (calculated in a $2 \mathrm{D}$ approximation) of the merge element due to the voltages on the rods of $Q D 5$. 
TABLE I. Typical quadrupole operating voltages. All the quadrupoles have four cylindrical electrodes per beam channel, except for $Q 4$ and $Q D 5$. $Q 4$ has hyperbolic-shaped electrodes. $Q D 5$, a combined dipole plus quadrupole, has the voltage distributed to the 71 individual tungsten electrodes via a resistive divider.

\begin{tabular}{cc}
\hline \hline Quadrupole & Voltage $(\mathrm{kV})$ \\
\hline$Q 1$ & 4.756 \\
$Q 2$ & 3.880 \\
$Q 3$ & 3.878 \\
$Q 4$ & 2.932 \\
$Q D 5$ & \pm 3.80 \\
$Q 6$ & 8.94 \\
$Q 7$ & 14.66 \\
$Q 8$ & 15.99 \\
$Q 9$ & 16.53 \\
$Q 10$ & 17.75 \\
$Q 11$ & 19.31 \\
$Q 12-Q 67$ & 20.0 \\
\hline \hline
\end{tabular}

via a pair of power supplies $( \pm 5 \mathrm{kV})$ and a resistive divider. The cage design of $Q D 5$ provides the dipole and quadrupole fields in the required short axial distance and also shields the beams' (nonlinear) space charge from one another.

Beam steering is accomplished with sources mounted on gimbals which allow them to rotate about their centers, followed by articulation in $x$ and $y$ of $Q 2$. Both operations can be done in vacuum. Since the beams emerging from QD5 are separated by about $4 \mathrm{~mm}$ (beam edge to beam edge), their clearance from the rods within the wire cage is only $\sim 1.5 \mathrm{~mm}$ near the exit of the cage. Thus, steering must be accurate to better than $1 \mathrm{~mm}$ in order to minimize the emittance growth while avoiding sampling the nonlinear fields of the wire cage that are significant within $\sim 1 \mathrm{~mm}$ (i.e., one rod diameter) of the tungsten electrodes.

Regarding the design of a combined-function (quadrupole and dipole) element at the higher beam kinetic energy $(\sim 100 \mathrm{MeV})$ suitable for a driver, a magnetic element is preferred to an electrostatic element. The voltage required in an electrostatic element would be $\sim 50 \mathrm{kV}$, and breakdown initiated by small fractional beam loss might prevent reliable operation. A roomtemperature magnetic merging element with a thin (few $\mathrm{mm}$ ) iron yoke in the tangency region between the beams (but a thicker yoke outside the group of four beams) would carry the flux in that crucial area.

The first beam diagnostic station $(d 1)$ is located in the drift regions between $Q 2$ and $Q 3$, the second ( $d 2)$ between $Q 4$ and $Q D 5$, and the third (d3) between $Q 7$ and $Q 8$. Subsequent diagnostic stations $(d 4-d 9)$ are in drift spaces after groups of ten quadrupoles through the rest of the MBE-4 transport channel. Diagnostics $d 1$ and $d 2$ have two-slit emittance scanners for each trans- verse dimension for each beam. (Attached to the downstream slit of each pair is a Faraday cup which measures the transmitted beam.) Each slit assembly is independently driven by a computer controlled step motor, with a positioning accuracy of $\sim 10 \mu \mathrm{m}$. The step motors reside outside the vacuum system and drive the diagnostic slit (or slit and Faraday cup) via a ferrofluidic seal and lead screw assembly. The width of the slits in the scanning direction is 50,100 , or $200 \mu \mathrm{m}$, depending on the station, and each slit and its corresponding slit-Faraday cup are separated longitudinally by $10 \mathrm{~cm}$. The downstream diagnostic stations also include Faraday cups for total beam current measurements. The beam currents from the four diodes were periodically measured with Faraday cups in place of the $Q 2$ quadrupole array $(2.6 \mathrm{~mA} /$ beam $)$.

In addition to beam profiles and phase space measurements in each of the transverse planes, measurements of the 2-dimensional current density profile $J(x, y)$ were inferred from measurements using "crossed slits," or one $x$ slit with a $y$ slit. The beam energy and dc quadrupole voltages were stable from pulse to pulse so that phase space was measured by sampling the phase space distribution density with $20-200$ beam pulses (at $\sim 0.3 \mathrm{~Hz}$ ), one pulse for each $x, y$ ( $x, x^{\prime}$ or $y, y^{\prime}$ depending on the diagnostic) coordinate sampling location.

The pressure in the experiment was highest near the contact-ionization $\mathrm{Cs}^{+}$sources and their graphite Pierce electrodes ( $P \approx 3-4 \times 10^{-6}$ Torr), which were a considerable source of outgassing when heated (the base pressure with the sources cold was $<1 \times 10^{-6}$ Torr). Downstream of $d 3$, the pressure during beam measurements was $\approx 2 \times 10^{-7}$ Torr.

\section{RESULTS}

\section{A. Individual beams before merging}

The multiple beam induction-linac designs for heavy ion fusion rely on each of the beams having very similar beam properties, because it is envisioned that neighboring quadrupole channels of a multiple beam array will have nearly identical gradient and field quality. Individual (dipole) correction elements every several lattice periods will maintain individual beam centroid alignment to avoid having the beam sample the higher order multipoles of the quadrupoles and to avoid scraping of the beam along the wall of the beam line.

In this experiment there are two diagnostic stations $(d 1, d 2)$ upstream of the merge element (QD5). Measurements at these stations were principally used to verify the initial beam parameters and to steer the beams' centroids using the angle adjustments of the source diodes and transverse displacements of the $Q 2$ quadrupoles for each beam. To illustrate the similarity between the beams in this experiment, the profiles of the four beams measured at $d 1$ are overlaid for comparison in Fig. 3. The $2 \mathrm{rms}$ beam radii of the beams were identical to within 


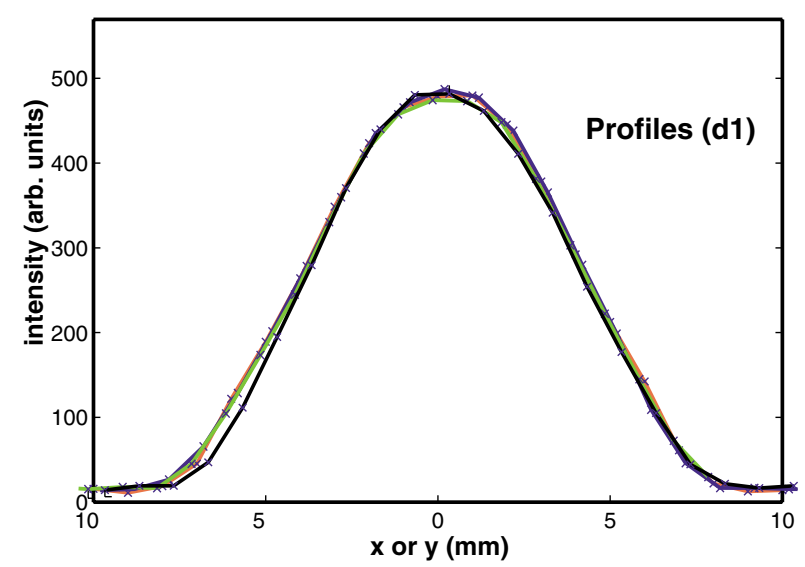

FIG. 3. (Color) Transverse beam profiles measured at the $d 1$ diagnostic station. The rms beam radii are identical to within $\pm 0.2 \mathrm{~mm}$.

$\pm 0.2 \mathrm{~mm}$ of their average of $6.5 \mathrm{~mm}$, and the individual beam currents were $2.5<I<2.7 \mathrm{~mA}$. The measured initial normalized emittance of the individual beams at $d 1$ was $\varepsilon(1)_{n}=0.025 \mathrm{~mm}$ mrad. The measured beam parameters at $d 1$ were used to initialize the particle-in-cell (PIC) simulations. The initial perveance was $K_{i}=6.3 \times$ $10^{-4}$. The beam envelope is described by the rms envelope equation:

$$
\frac{d^{2} a}{d s^{2}}= \pm \kappa_{x} a+\frac{\varepsilon_{x}^{2}}{a^{3}}+\frac{K}{(a+b)}
$$

where $a$ and $b$ are the horizontal and vertical semimajor and minor axes of the envelope, $\varepsilon_{x}$ is the emittance, and $\kappa_{x}$ is the focusing constant. The ratio of the emittance to the space-charge term for the initial beams, $\approx \varepsilon^{2} /(2 K a) \sim 10^{-2}$, illustrates the relative importance of space charge in this experiment.

HIBEAM, a 2D PIC code written to simulate the transverse dynamics of high space-charge beams propagating in electrostatic focusing lattices, was used for most numerical simulations of this experiment [15]. Cross-checks were made with runs through $Q 4$ using the $3 \mathrm{D}$ PIC code WARP [16], and validated the accuracy of the 2D approximation. The applied and image fields were solved with a fast-Fourier transform and capacity matrix. The transverse grid cell size was $0.2 \times 0.2 \mathrm{~mm}$, while the average beam-envelope radius ranges from $\approx 6 \mathrm{~mm}$ (upstream of $Q D 5$ ) to $\approx 15 \mathrm{~mm}$ (downstream). The number of macroparticles was 4096 and the longitudinal steps size was $5 \mathrm{~mm}$. The initial distribution for the simulation was semi-Gaussian, with an initial envelope ( $a=b=3 \mathrm{~mm}$, $a^{\prime}=b^{\prime}=-2 \mathrm{mr}$ ), emittance [ $\varepsilon(1)_{n}=0.025 \mathrm{~mm} \mathrm{mrad}$, and beam current $(2.6 \mathrm{~mA})$ based on measurements at $d 1$. $Q 1-Q 3$ and $Q 6-Q 67$ were modeled with cylindrical focusing electrodes. $Q 4$ was modeled with hyperbolic surfaces as in the experiment. The tapered geometry [17] of $Q D 5$ was modeled by dividing the rod lengths into 16 equal segments parallel to the centerline of the combiner, but with an aperture equal to that of the actual wire cage at the center of the interval.

The transverse phase space of one of the beams at $d 2$ is shown in Fig. 4, along with the same view as calculated by the PIC simulations. At $d 1$, the phase space measurements and the simulations showed less distortions than at $d 2$. At $d 2$, some distortions are apparent in the data (and simulations) due to the compression near $Q 4$ of the initially semi-Gaussian distribution. The distortion arises because the semi-Gaussian initial state in the simulation (which reasonably represents the initial state distribution in the experiment) is not an equilibrium distribution. The thermal velocity distribution subjects
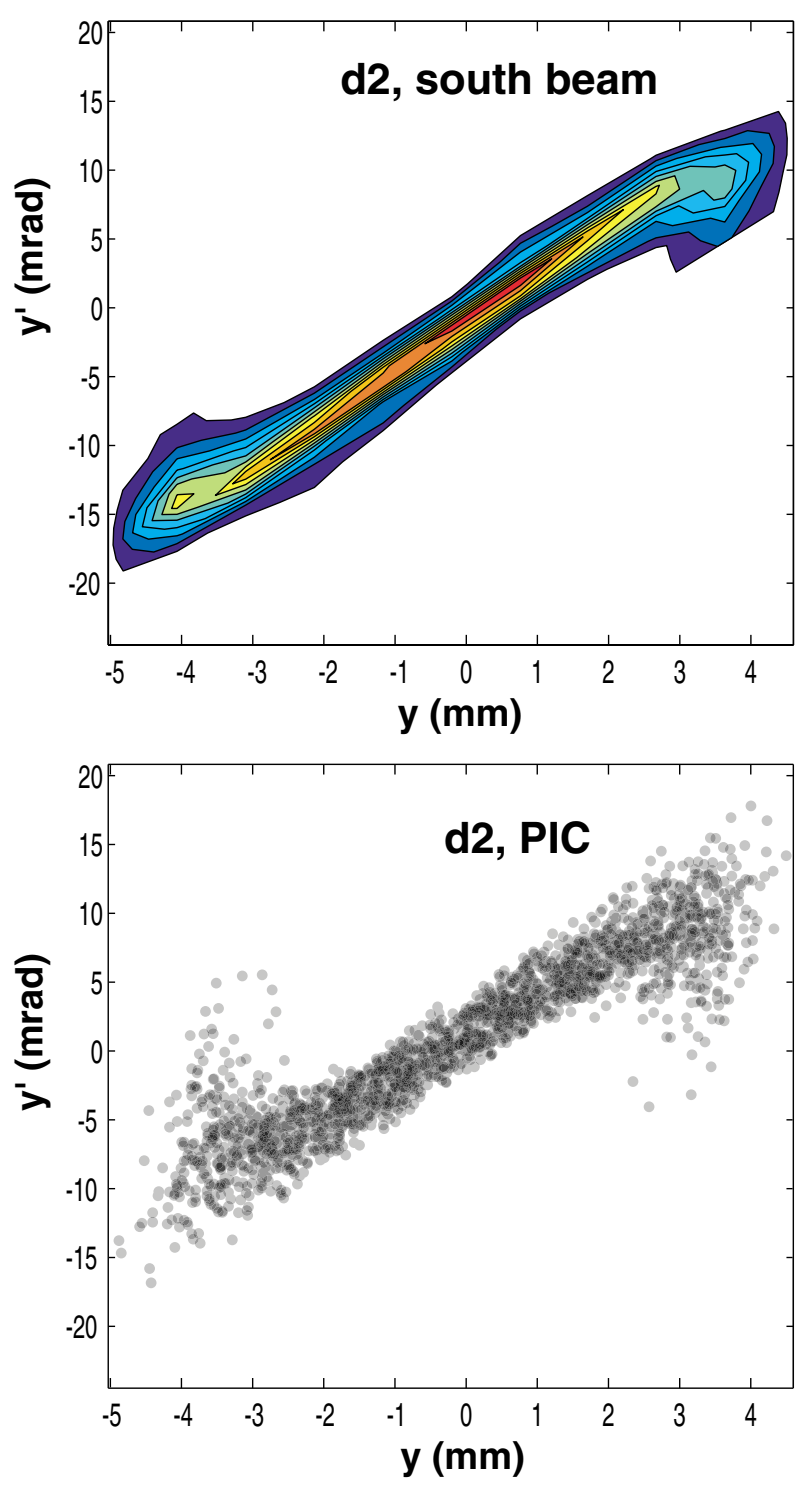

FIG. 4. (Color) Measured transverse phase space at $d 2$ of one beam compared to the result from PIC simulations. The contour lines indicate $10 \%$ increments in relative intensity, and the color code sequence from lowest to highest intensity is blue, ..., red. 
particles at the beam edge to a nonlinear kick from space charge and consequently a larger phase advance. Compared to earlier measurements [15] the effect is less pronounced because of modifications to the matching solution that lessen the beam-envelope aspect ratio $(a: b)$ between the diodes and QD5. Adjustments to the angles of each source and the transverse displacement of each $Q 2$ quadrupole position the beams to within $0.5 \mathrm{~mm}$ and $0.5 \mathrm{mr}$ of the desired location and angle at $d 2$, just upstream of the combined-function element $Q D 5$. In the drift region between $Q 4$ and $Q D 5$ the beams are $\approx 3 \mathrm{~cm}$ apart, and the electrostatic beam-beam repulsion deflects the beams by a few milliradians in this $11 \mathrm{~cm}$ gap.

\section{B. Measurements of the merged beam}

In early stages of the experiment, higher current $(4.5 \mathrm{~mA})$ beams were injected and merged. For this higher injected current $(1.65 \times$ design value), it was not possible to match the cylindrically symmetric beams from the diodes to the desired envelope parameters at the exit of $Q D 5$ without scraping and beam loss at $Q 4$. Scraping was avoided allowing the beams to exit the cage with nonzero divergence ( $\approx 10 \mathrm{mrad}$ ), consequently, the phase space occupied by the beams was increased by a factor of 2 and approximately $88 \%$ of the injected current was transmitted to $d 3$. The phase space measurements were qualitatively understood, but the larger than expected beam loss was not. In order to deliver the design current of $\approx 2.6 \mathrm{~mA} /$ beam, new Pierce electrodes masked the periphery of the ion emitters. (Aperturing masks between each diode exit and $Q 1$ would have allowed merging measurements at several initial beam currents, but phase space measurements at $d 1$ showed nonuniform beam profiles and large convergence angles that precluded proper matching downstream.)

The merged beam current at $d 3$ was $10.2 \pm 0.1 \mathrm{~mA}$ or $98 \%$ of the injected beam current $(4 \times 2.6 \mathrm{~mA})$. During the course of the experiment, the matching solution of the merged beams to the downstream transport lattice was improved to reduce the beam edge-to-wall clearance in the first several quadrupoles $(Q 6-Q 11)$. The first diagnostic station after the merge point, $d 3$, is one quadrupole doublet downstream of $Q D 5$. Adjustments to the gradients of $Q 6$ and $Q 7$ resulted in smaller maximum envelope excursions in those quadrupoles (from 21 to $19 \mathrm{~mm}$; the physical aperture is $R=27 \mathrm{~mm}$ ), and rms envelope parameters at $d 3$, with similar convergence and divergence angles $\left(a^{\prime} \approx-b^{\prime}\right)$ and similar beam semimajor and semiminor axes $(a \approx b)$. These three effects made the merged beam easier to match to the downstream transport lattice using the $Q 8-Q 11$ quadrupoles.

We found that because the dynamics of the transverse phase space are complex (due to the nonuniform current density) near $d 3$, initializing envelope calculations with the $d 3$ data for the purpose of matching the envelope to the downstream lattice was less accurate and resulted in higher beam loss due to scraping than was ultimately achieved. The horizontal and vertical beam phase spaces at $d 3$ are shown in Figs. 5 and 6, where the individual beams can be distinguished from each other. Ultimately, improvements to the downstream match were made by measuring the phase space at $d 4$, where the merged beam was nearer equilibration. The extracted rms envelope
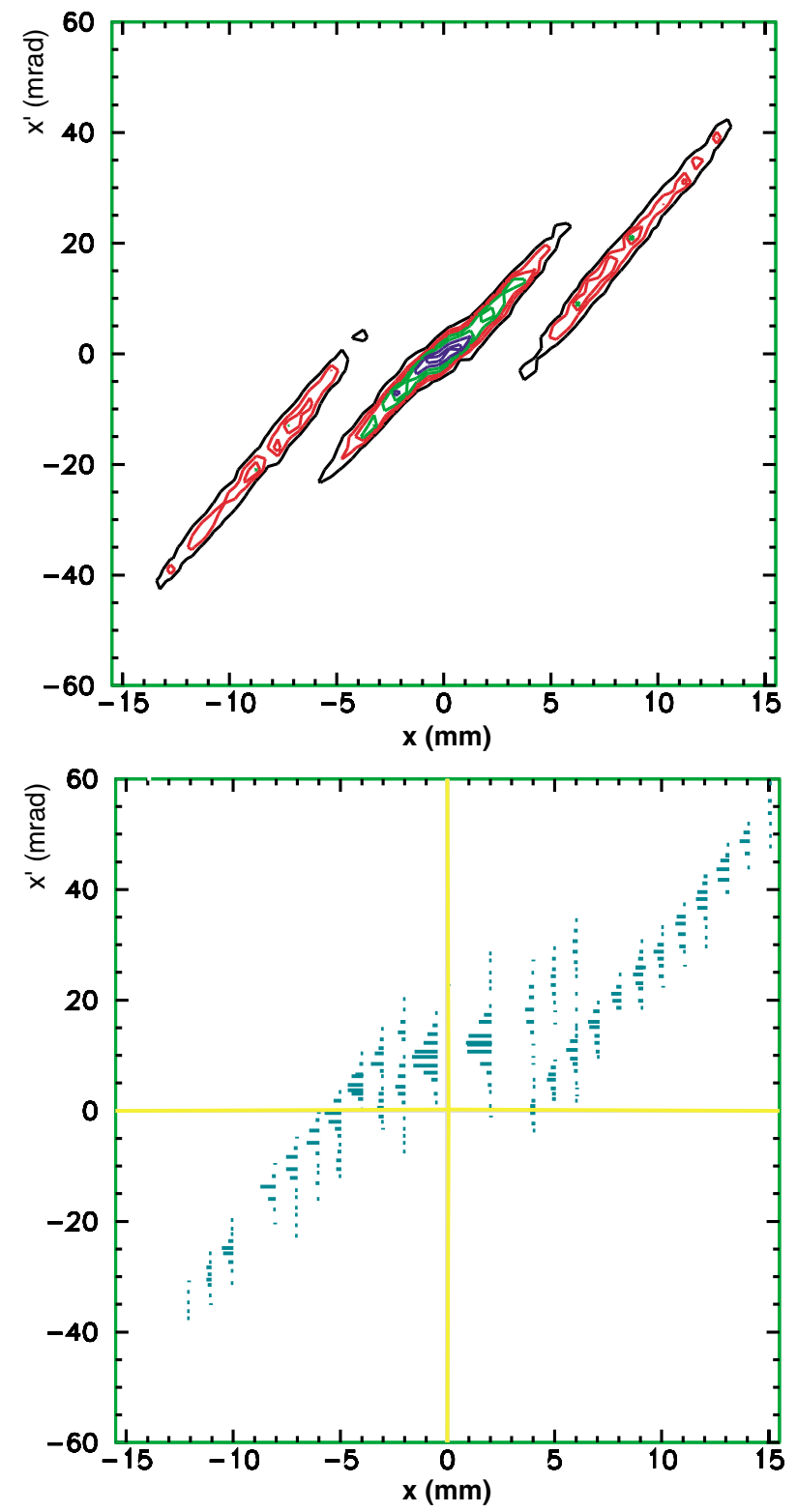

FIG. 5. (Color) Horizontal phase space measurement (bottom panel) versus PIC simulation (top panel) at the $d 3$ diagnostic station. For the data, the length of the horizontal bar indicates the signal amplitude measured for the phase space coordinate at the right edge of each bar. For the simulation, the central group of contour lines ( $10 \%$ intervals) includes the distribution of two of the four merged beams in this projection of phase space. 

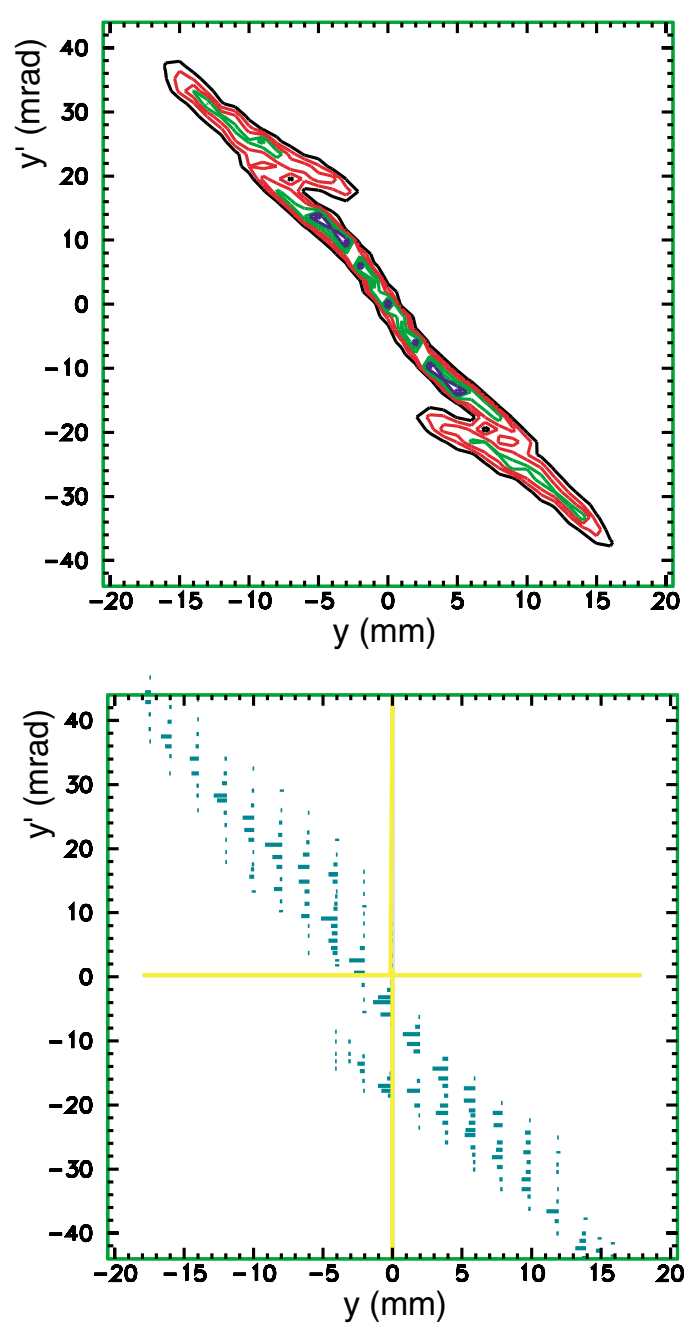

FIG. 6. (Color) Vertical phase space measurement (bottom panel) versus PIC simulation (top panel) at the $d 3$ diagnostic station. For the data, the length of the horizontal bar indicates the signal amplitude measured for the phase space coordinate at the right edge of each bar. For the simulation (10\% contour intervals), the higher density region near $y=0, y^{\prime}=0$ includes the distribution of two of the four merged beams in this projection of phase space.

parameters were used to constrain the upstream quadrupole gradients by integrating the envelope equation backwards (from $Q 17$ through $Q 8$ ) to revise the $Q 8-Q 11$ quadrupole voltages. This iterative process for setting the $Q 8-Q 11$ voltages led to a better envelope match of the merged beam to the lattice at $d 4(Q 18)$ and beyond. If research accelerators were to rely on beam merging, then based on this experience, extensive diagnostics in the downstream region within a few beam plasma periods of the merge point would improve envelope matching and minimize beam loss.

The 2D transverse intensity map at $d 3$ was measured with a 1-mm slit oriented at $90^{\circ}$ to a slit and Faraday cup $10.0 \mathrm{~cm}$ downstream. Because of the $10-\mathrm{cm}$ separation between the crossed slits the resulting (astigmatic) image differs from an intensity map at a fixed axial location: The ribbon of beam passing from the first to the second slit is converging (due to the upstream focusing lattice) and this effect, including space charge, was incorporated into the PIC simulation. The beam was mapped in 1-mm steps in both the $x$ and the $y$ directions and the resulting contour plots from the measurement and the PIC simulation are shown in Fig. 7. The overall perimeter of the beam is fairly well reproduced by the PIC simulation, along with the qualitative features of the main intensity variations within. At this diagnostic station, the merged
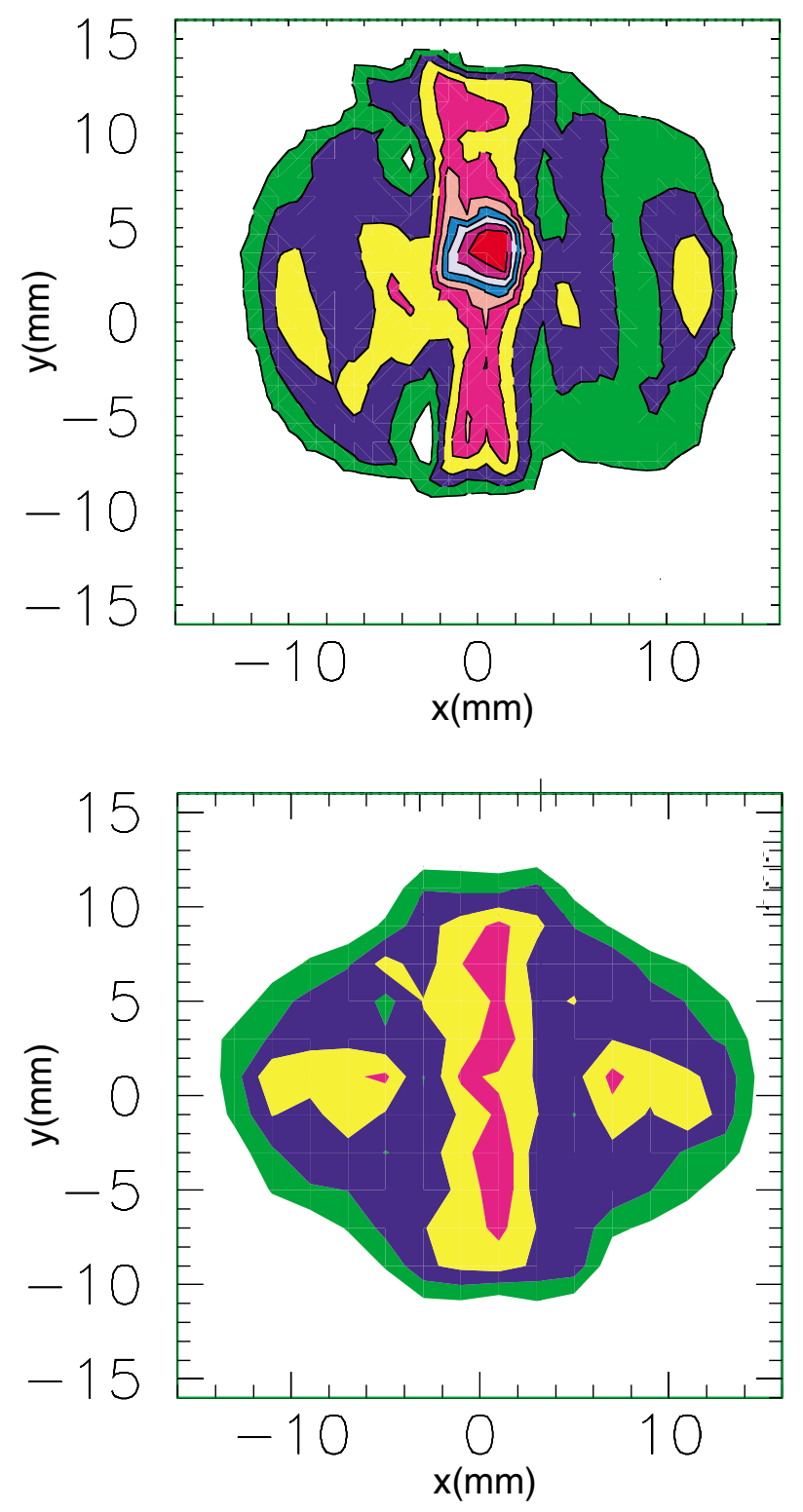

FIG. 7. (Color) Data (top panel) compared to PIC simulation (bottom panel) of the crossed-slit measurement at the $d 3$ diagnostic station. The contour lines for the experimental data indicate $10 \%$ increments in relative intensity (20\% contours for the PIC simulation) and the color code sequence from lowest to highest intensity is green, blue, yellow, pink,..., red. 
beam is far from equilibrated: It is only 0.15 plasma periods $\left(2 \pi v_{z} / \omega_{p}\right)$ following the merge point. Thus, individual beams are resolved in the phase space distributions measured at $d 3$. These measurements show that some residual misalignments of the beamlets' centroids remain, predominantly in the horizontal plane. The effect of these misalignments was explored in the PIC simulations and is discussed below.

At the $d 5$ diagnostic, 22 quadrupoles or $\approx 2 \lambda_{p}$ downstream of the merge point, there is qualitative agreement between the simulations and the measurements (Figs. 8-10). The simulations and data indicate that the phase mixing of the very nonuniform phase space distri-

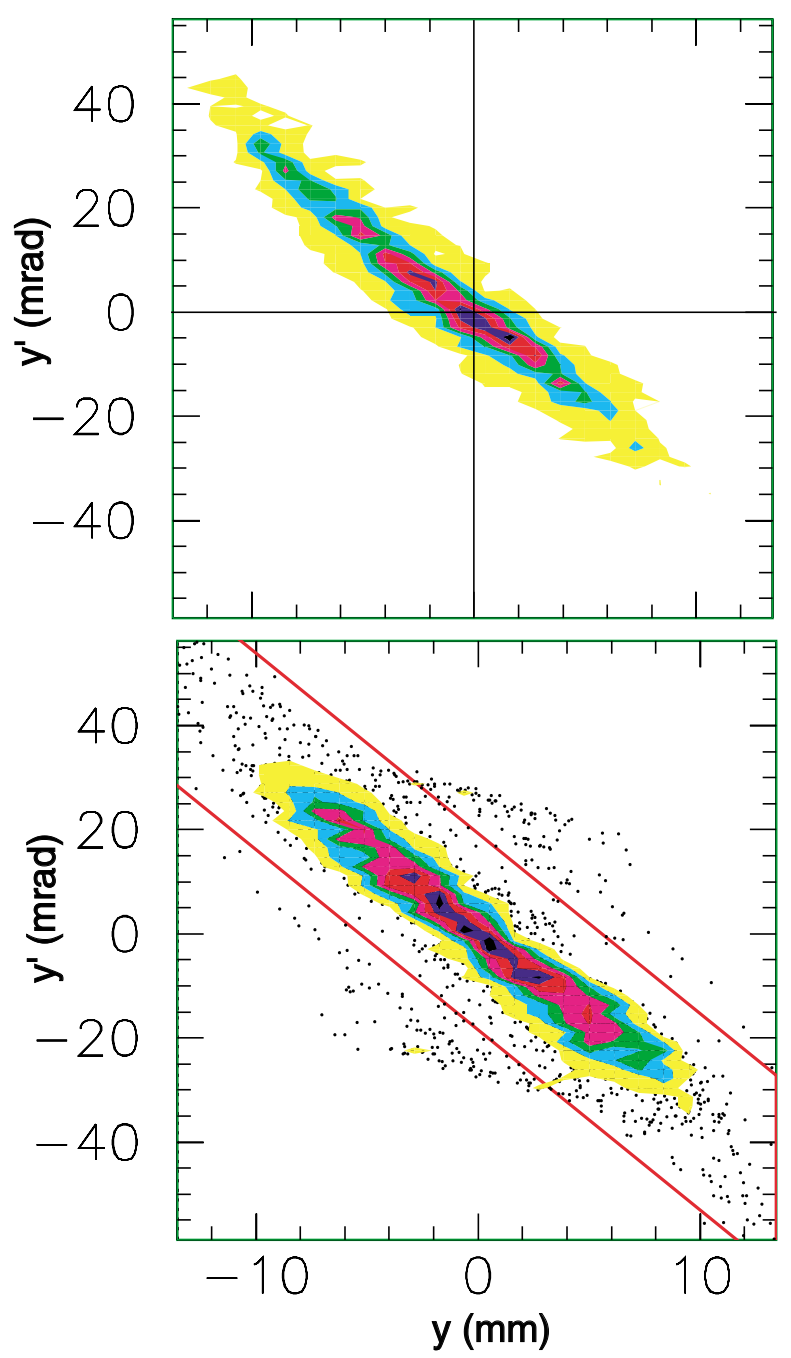

FIG. 8. (Color) Experimentally measured vertical phase space (top panel) versus PIC (bottom panel) at $d 5$. The red box indicates for the simulation the region inside of which particles were included for the PIC (88\%) emittance calculations of Table II. The contour lines indicate $12.5 \%$ increments in relative intensity, and the color code sequence from lowest to highest intensity is yellow,...black. All of the PIC particles that lie outside of the lowest contour level are shown in order to illustrate the details of the calculated halo. bution that was evident at $d 3$ (Figs. 5-7) has advanced to the point that the individual beams are no longer resolved. The envelope parameters (see Table II) measured in the experiment are within $10 \%-20 \%$ of the PIC simulation, with better agreement in the vertical $\left(b, b^{\prime}\right)$ plane. These differences are greater than the sensitivity of the diagnostics and the overall stability of the system (Marx voltage, quadrupole voltages). Repeated measurements of the beam centroid and envelope at $d 5$ reproduce to

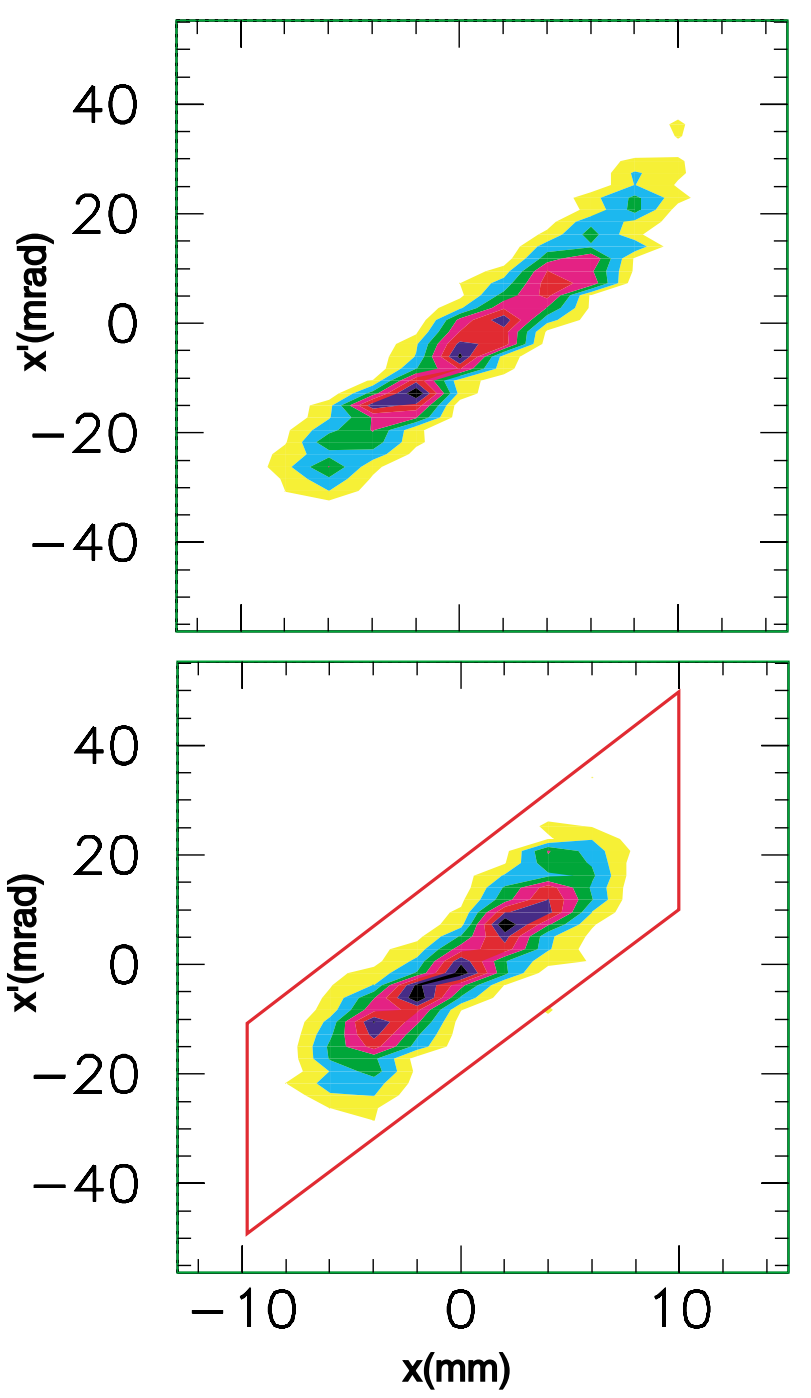

FIG. 9. (Color) Horizontal phase space measurements (top panel) at $d 5$ compared to the PIC simulation (bottom panel). The contour lines indicate $12.5 \%$ increments in relative intensity, and the color code sequence from lowest to highest intensity is yellow,...black. The red box indicates for the simulation the region inside of which particles were included for the PIC (88\%) emittance calculations of Table II, and the interior is approximately the same as the scanned boundary in the experiment. Approximately 7\% of the PIC particles lie outside of this box. An additional 5\% of the initial particles are lost in the simulation upstream when the particles strike a focusing electrode. 

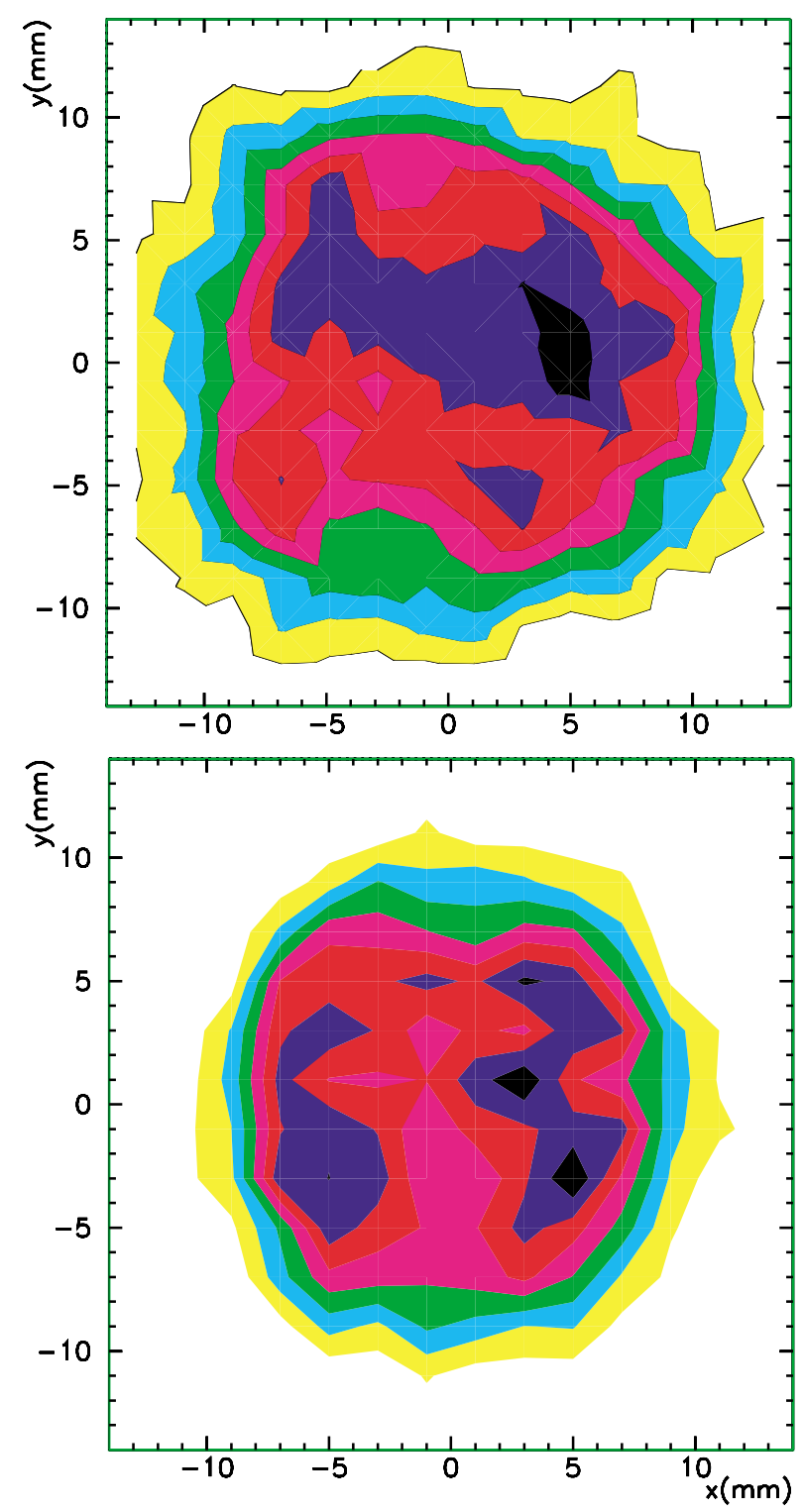

FIG. 10. (Color) Data (top panel) compared to PIC simulation (bottom panel) of the crossed-slit measurement at the $d 5$ diagnostic station. The contour lines indicate $12.5 \%$ increments in relative intensity, and the color code sequence from lowest to highest intensity is yellow,... black.

within $0.5 \mathrm{~mm}$. The measured merged beam centroid at $d 5$ was within $1.2 \mathrm{~mm}$ and $2.9 \mathrm{mrad}$ of the central axis of the channel. The beam current loss in the experiment is cumulatively $10 \%-15 \%$ at $d 5\left(85 \%<I / I_{0}<90 \%\right)$, while the PIC simulations predict 5\% loss. This current difference might be responsible for much of the discrepancy in the envelope parameters. For the calculation of the rms parameters in the PIC (95\%) case, the rms parameters and emittance were calculated for all of the surviving particles at $d 5$ (95\% of the initial number of particles loaded). For the PIC (88\%) case an additional $7 \%$ of the surviving particles of the PIC (outside the red boxes of Figs. 8 and 9) were excluded for the calculation of the rms
TABLE II. Comparison of experimentally observed beam envelope, centroid, current (from Faraday cup data), and emittance at $d 5$ to the PIC simulation.

\begin{tabular}{lcccccc}
\hline \hline & $\begin{array}{c}a \\
(\mathrm{~mm})\end{array}$ & $\begin{array}{c}a^{\prime} \\
(\mathrm{mrad})\end{array}$ & $\begin{array}{c}b \\
(\mathrm{~mm})\end{array}$ & $\begin{array}{c}b^{\prime} \\
(\mathrm{mrad})\end{array}$ & $\begin{array}{c}I / I_{0} \\
\%\end{array}$ & $\begin{array}{c}\varepsilon_{n} \\
(\mathrm{~mm} \mathrm{mrad})\end{array}$ \\
\hline Experiment & 10.2 & 32 & 10.9 & -36 & $85-90$ & 0.19 \\
PIC (95\%) & 8.1 & 32.6 & 11.0 & -37.6 & 95 & 0.30 \\
PIC (88\%) & 8.0 & 28.8 & 10.2 & -37.0 & 88 & 0.20 \\
\hline \hline
\end{tabular}

envelope parameters and emittance, and the $I / I_{0}$ is in the middle of the range observed in the experiment. The agreement is not perfect, and discrepancies between the calculated and measured distributions are due in part to inconsistencies in the initial PIC distribution (semiGaussian), quadrupole alignment offsets, and binning (simulation) and sampling (experiment) intervals.

The evolution of the rms emittance calculated from the PIC simulations is plotted in Fig. 11. Upstream of the merging element $(Q D 5)$ the emittance of one of the four beams is plotted, and downstream, the emittance of the four-beam ensemble is plotted. The calculation, initialized with a semi-Gaussian distribution using the measured emittance and current at $d 1$, predicts a rapid rise in emittance following the merging element. The initial increase in the first few plasma periods after $Q D 5$ is due largely to the space-charge nonlinearity. As an indication of the importance of space-charge free energy, we plot the emittance from the analytically tractable

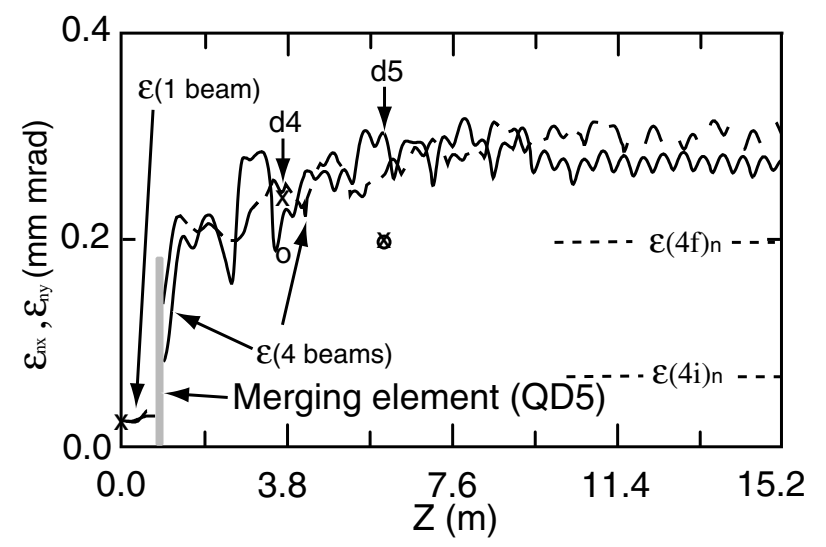

FIG. 11. Evolution of emittance from the PIC simulation (dashed line for $\varepsilon_{n x}$, solid line for $\varepsilon_{n y}$ ). The emittance is calculated for all surviving particles in the simulation $(95 \%$ at $d 5)$. Upstream of the merging element (QD5), the emittance of one of the four (2.6 mA) identical beams is shown. Downstream of the merging element, the emittance of the $\approx$ $10 \mathrm{~mA}$ merged beam is shown. The locations of the $d 4$ and $d 5$ diagnostic stations are indicated. The $d 5$ station is at the end of the region of rapid emittance growth. The points below the curve are for the experimental data at $d 4$ and $d 5$ (cross $=\varepsilon_{n x}$, circle $=\varepsilon_{n y}$ ). The values $\varepsilon(4 i)_{n}$ and $\varepsilon(4 f)_{n}$ are from the analytical model described in the text. 
TABLE III. Initial normalized emittance before merging and merged beam emittance from an analytical model.

\begin{tabular}{ccc}
\hline \hline $\begin{array}{c}\varepsilon(1)_{n} \\
(\mathrm{~mm} \mathrm{mrad})\end{array}$ & $\begin{array}{c}\varepsilon(4 i)_{n} \\
(\mathrm{~mm} \mathrm{mrad})\end{array}$ & $\begin{array}{c}\varepsilon(4 f)_{n} \\
(\mathrm{~mm} \mathrm{mrad})\end{array}$ \\
\hline 0.025 & 0.067 & 0.20 \\
\hline \hline
\end{tabular}

model of merging four round, uniform current density beams [6]. First, note that emittance growth occurs when the four beams first "see" each other at the exit of the combiner $(Q D 5), \varepsilon(4 i)_{n}$ in Fig. 11. This is the emittance growth due to phase space dilution in the low intensity limit (negligible space charge). The analytic model also predicts that the final emittance of the merged beam $\varepsilon(4 f)_{n}=2.8 \varepsilon(4 i)_{n}$ has a dominant contribution from conversion of the free space-charge field energy of the initial merged beam distribution (Fig. 2 and Table III). Note that compared to the rapid increase in the first few meters after the merge, the PIC simulation predicts a relatively slow emittance growth downstream, and likewise, the experimental data show little emittance growth between $d 4$ and $d 5$.

The PIC emittance in Fig. 11 is calculated for all surviving particles in the simulation. Particle loss in the simulation is small ( $<0.5 \%$ ) at $d 4$, but increases to $5 \%$ at $d 5$. Since the phase space diagnostic does not detect all surviving particles in the aperture, the calculated emittance is larger than the measured emittance; this difference is mostly understood (see discussion of Figs. 8 and 9, Table II). The uncertainty on the measured emittance $(\approx \pm 10 \%)$ is mainly due to diagnostic slit width and its relative alignment.

\section{Effect of beam centroid alignment errors}

At $d 3$, the beam is measured to have offsets of $\sim 1-2 \mathrm{~mm}$ and several milliradians. This is the overall offset arising from the individual misalignments of the four beams at the exit of $Q D 5$. The influence of these offsets on the phase space evolution and beam loss was studied in the PIC simulation by executing a run with initial beams that are aligned perfectly. Then the particle coordinates (for each of the macroparticles) were shifted by the experimentally observed centroid offsets in $x, x^{\prime}, y$, and $y^{\prime}$ coordinates. This was done in two ways: First, the experimentally observed centroid offset of the $10.2 \mathrm{~mA}$ merged beam was applied to the PIC distribution particles at the $d 3$ location. This produced little change in the PIC particle loss at $d 5$, and the emittance was $\approx 2 \%$ greater than for a simulation with perfect alignment. In the second approach, centroid offsets were estimated for each of the four beamlets, still resolved in the $d 3$ data (see Figs. 5 and 6) and applied to the individual PIC beams at the $d 3$ location. Similar to the first test, this produced a negligible effect on calculated beam loss and the emittance was increased by $\approx 5 \%$. A parametric study of beam loss versus centroid offset showed that a transverse displacement of the beam at $d 3$ by $3-4 \mathrm{~mm}(>2 x$ of the experimentally observed offset) would be required to increase the beam loss from $5 \%$ to $6 \%$ at $d 5$. Thus the beam centroid control was sufficient.

The PIC simulations are able to account for roughly half of the observed beam loss. Some effects are very difficult to include realistically in the simulations. For instance, an additional $2 \%-3 \%$ of the beam particles are lost from beam-background gas collisions based on the pressure measured in the experiment. Further losses due to halo ions scraping and desorbing neutral atoms that interact with the beam later in the pulse are difficult to model due to large uncertainties in the desorbed atom dynamics and the relevant atomic cross sections.

\section{CONCLUSIONS}

In a scaled experiment to explore a merging beam concept that would improve the cost effectiveness of a HIF driver, we have found the concept viable. The 4:1 merging would allow for a shorter initial pulse length in the driver and a larger aperture fill factor in the low energy end of the accelerator. The magnitude of the emittance growth measured in the experiment is understood to be due in large part to the conversion of spacecharge free energy to emittance, and the magnitude of this increase is consistent with an analytical model and particle in cell simulations. Both the numerical simulations and the experiment show relatively slow emittance growth a short distance after the merge.

The experimentally observed $10 \%-15 \%$ beam loss in the experiment is due partly to beam-background gas interaction and envelope mismatch. The simulation predicts 5\% beam loss and neglects all beam-gas interactions. The beam loss would be mitigated with lower vacuum system pressure and more extensive diagnostics to measure and rematch the beam after the merge point.

The results indicate that for some HIF driver designs, the phase space dilution from merging may be acceptable. A merging experiment at higher beam kinetic energy and beam intensity would be a valuable next step in addressing particle loss, beam diagnostics, and merging element functionality closer to the scale of interest. The best driver design will depend on the evolution of target design, driver system optimization, and results such as those presented here, which will allow a rational evaluation of the merging option. The main intent of this experiment, to understand the beam combining process, was successful.

\section{ACKNOWLEDGMENTS}

The authors gratefully acknowledge the outstanding support of the LBNL technical staff including Mr. R. Hipple and Mr. W. Strelo. W. Fawley and K. Hahn provided support for the HIBEAM simulations. I. Haber 
(NRL), David Grote (LLNL), and David Judd also provided support and insight. This work was supported by the Director, Office of Energy Research, Office of Fusion Energy, U.S. DOE, under Contract No. DE-AC0376SF00098.

[1] A. Schempp, Nucl. Instrum. Methods Phys. Res., Sect. A 415, 209 (1998).

[2] J. E. Stovall, F.W. Guy, R. H. Stokes, and T. P. Wangler, Nucl. Instrum. Methods Phys. Res., Sect. A 278, 143 (1989).

[3] K. F. Johnson et al., Part. Accel. 37-38, 261 (1992).

[4] H. Zimmermann et al., in Proceedings of the 2001 Particle Accelerator Conference, Chicago, 2001, edited by P. Lucas (IEEE, Piscataway, NJ, 2001), p. 3918, http:// accelconf.web.cern.ch/AccelConf/p01/PAPERS/ FPAH103.PDF

[5] D. P. Grote, E. Henestroza, and J.W. Kwan, Phys. Rev. ST Accel. Beams 6, 014202 (2003).

[6] C. M. Celata et al., in Proceedings of the 1987 Particle Accelerator Conference, Washington, DC, 1987, edited by E. Lindstrom and L. Taylor (IEEE, Piscataway, NJ, 1987), p. 1167.
[7] O. A. Anderson, Fusion Eng. Des. 32-33, 209 (1996).

[8] P. A. Seidl et al., Nucl. Instrum. Methods Phys. Res., Sect. A 415, 243 (1998).

[9] P. A. Seidl et al., in Proceedings of the Particle Accelerator Conference, Vancouver, Canada, 1997, edited by M. Comyn et al. (IEEE, Piscataway, NJ, 1997), p. 1926.

[10] W. M. Fawley et al., in Proceedings of the Particle Accelerator Conference, Vancouver, Canada, 1997 (Ref. [9]), p. 1923.

[11] R. O. Bangerter, Philos. Trans. R. Soc. London, Ser. A 357, 575 (1999).

[12] R. O. Bangerter, Nucl. Instrum. Methods Phys. Res., Sect. A 464, 17 (2001).

[13] J. J. Barnard et al., Nucl. Instrum. Methods Phys. Res., Sect. A 415, 218 (1998).

[14] Thomas J. Fessenden et al., in Proceedings of the 1987 Particle Accelerator Conference, Washington, DC, 1987, (Ref. [6]), p. 898.

[15] W. M. Fawley et al., in Proceedings of the Particle Accelerator Conference, Vancouver, Canada, 1997 (Ref. [9]), p. 1923.

[16] D. P. Grote et al., Nucl. Instrum. Methods Phys. Res., Sect. A 415, 428 (1998).

[17] C. M. Celata et al., Fusion Eng. Des. 32-33, 219 (1996). 